\title{
Small bipartite subgraph polytopes
}

\author{
Laura Galli ${ }^{\mathrm{a}}$, Adam N. Letchford ${ }^{\mathrm{b}, *}$ \\ a University of Bologna, Italy \\ ${ }^{\mathrm{b}}$ Department of Management Science, Lancaster University, Lancaster LA1 4YW, United Kingdom
}

\section{A R T I C L E I N F O}

\section{Article history:}

Received 24 March 2010

Accepted 5 May 2010

Available online 9 June 2010

Keywords:

Polyhedral combinatorics

Max-cut problem

Integrality ratios

\begin{abstract}
A B S T R A C T
We compute a complete linear description of the bipartite subgraph polytope, for up to seven nodes, and a conjectured complete description for eight nodes. We then show how these descriptions were used to compute the integrality ratio of various relaxations of the max-cut problem, again for up to eight nodes.
\end{abstract}

(c) 2010 Elsevier B.V. All rights reserved.

\section{Introduction}

Let $G=(V, E)$ be an undirected graph, and let us call an edge set $F$ bipartite if the subgraph $G_{F}=(V, F)$ is bipartite. The bipartite subgraph polytope of $G$, which we will denote by $\operatorname{BIP}(G)$, is the convex hull of the incidence vectors of the bipartite edge sets [1]. Since a graph is bipartite if and only if it does not contain an odd circuit, $\operatorname{BIP}(G)$ is the convex hull of the vectors $x \in\{0,1\}^{|E|}$ satisfying the following inequalities:

$$
\sum_{e \in C} x_{e} \leq|C|-1 \quad \text { (for all circuits } C \subset E:|C| \text { odd). }
$$

These inequalities, known as odd circuit inequalities, were shown to define facets of $\operatorname{BIP}(G)$ in [1]. Further results on $\operatorname{BIP}(G)$ can be found in $[3,14]$.

The main motivation for studying $\operatorname{BIP}(G)$ is that the well-known max-cut problem, in the special case of non-negative edge weights, is equivalent to maximising a linear function over $\operatorname{BIP}(G)$. Indeed, a set of edges defines a cut in $G$ if and only if it is bipartite and is not strictly contained in another bipartite edge set. In other words, $\operatorname{BIP}(G)$ is the submissive or downward monotonisation of the cut polytope $\operatorname{CUT}(G)$, which is the convex hull of the incidence vectors of the cuts in $G[2]$.

Now, let $\mathrm{BIP}_{n}$ and $\mathrm{CUT}_{n}$ denote the bipartite subgraph and cut polytopes, respectively, for the complete graph $K_{n}$. In principle, a complete linear description of $\operatorname{BIP}(G)$ or $\operatorname{CUT}(G)$ can be obtained from a complete description of $\mathrm{BIP}_{n}$ or $\mathrm{CUT}_{n}$, respectively, where $n=|V|$. This makes $\mathrm{BIP}_{n}$ and $\mathrm{CUT}_{n}$ worth studying.

\footnotetext{
* Corresponding author.

E-mail address: A.N.Letchford@lancaster.ac.uk (A.N. Letchford).
}

Since the max-cut problem is $\mathcal{N} \mathcal{P}$-hard [15], we cannot expect to obtain a complete description of $\mathrm{BIP}_{n}$ or $\mathrm{CUT}_{n}$ for general $n$. Complete descriptions of $\mathrm{CUT}_{n}$ have however been found for $n \leq 7$ [13], and a conjectured complete description has been computed also for $n \in\{8,9\}$ [5]. In fact, $\mathrm{CUT}_{n}$ has been explored in great depth; see, e.g., $[9,17]$ or $[19]$. The polytope $\mathrm{BIP}_{n}$, on the other hand, has received much less attention.

This paper makes two main contributions. First, we present a complete linear description of $\mathrm{BIP}_{n}$ for $n \leq 7$, and a conjectured complete description of $\mathrm{BIP}_{8}$, along with an explanation of how they were computed. In principle, these descriptions could lead to improved cutting-plane algorithms for max-cut instances with non-negative edge weights. Second, we show that the descriptions can be used to compute the exact integrality ratio of various relaxations of the max-cut problem, again with non-negative edge weights, for small values of $n$. Our hope is that this will shed light on the relative strengths and weaknesses of the relaxations.

The structure of the paper is as follows. In Section 2, we explain how we obtained the linear descriptions. In Section 3, we present an annotated list of facets. In Section 4, we define integrality ratios formally and explain how they can be computed. Finally, in Section 5, we present a table of integrality ratios, for various relaxations, along with a description of the max-cut instances for which the ratios are attained.

\section{Computing linear descriptions}

There exists a software package, called PORTA, which is capable of computing the facets of polyhedra of small dimension, given a list of the extreme points and extreme rays [4]. For $2 \leq n \leq 5$, we were able simply to generate all extreme points of $\mathrm{BIP}_{n}$ and feed them into PORTA, to obtain a complete linear description.

For $n \in\{6,7\}$, however, this naive approach did not work. This is simply because the number of extreme points is huge. (Indeed, 
even for $n=6$, there are thousands of extreme points.) To make progress for these values of $n$, we make use of the following lemma:

Lemma 1. Suppose an inequality induces a facet of $\mathrm{BIP}_{n}$, but is not equivalent to a non-negativity inequality $x_{e} \geq 0$ for some $e \in E$. Then it also induces a facet of $\mathrm{CUT}_{n}+\mathbb{R}_{-}^{\left(\begin{array}{c}n \\ 2\end{array}\right)}$, i.e., the Minkowski sum of the cut polytope and the non-positive orthant.

Proof. Let $\alpha^{T} x \leq \beta$ be an inequality that induces a facet of $\operatorname{BIP}_{n}$, and assume that it is not equivalent to a non-negativity inequality. Since $\mathrm{BIP}_{n}$ is the submissive of $\mathrm{CUT}_{n}, \alpha$ and $\beta$ are non-negative. Therefore, the inequality induces a facet also of $\mathrm{BIP}_{n}+\mathbb{R}_{-}^{\left(\begin{array}{c}n \\ 2\end{array}\right)}$. But $\mathrm{BIP}_{n}+\mathbb{R}_{-}^{\left(\begin{array}{c}n \\ 2\end{array}\right)}$ is equal to $\mathrm{CUT}_{n}+\mathbb{R}_{-}^{\left(\begin{array}{c}n \\ 2\end{array}\right)}$. So, the inequality induces a facet of the latter.

So, instead of enumerating all extreme points of $\mathrm{BIP}_{n}$ for $n \in$ $\{6,7\}$, we enumerated the extreme points of $\mathrm{CUT}_{n}$, which are far fewer, and provided them to PORTA, together with the $\left(\begin{array}{c}n \\ 2\end{array}\right)$ extreme

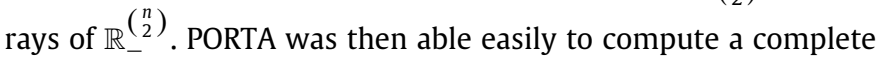
description of $\mathrm{CUT}_{6}+\mathbb{R}_{-}^{\left(\begin{array}{l}6 \\ 2\end{array}\right)}$. For $n=7$, it managed to compute the complete description, but only if we used the '-o' flag, which according to the PORTA manual invokes a heuristic for ordering the variables for Fourier-Motzkin elimination.

Once the descriptions of $\mathrm{CUT}_{n}+\mathbb{R}_{-}^{\left(\begin{array}{c}n \\ 2\end{array}\right)}$ were obtained, we organised the inequalities into equivalence classes, where two inequalities are deemed to be equivalent if one can be obtained from the other by a permutation of the nodes. We then took one inequality from each class, and checked whether it induced a facet of $\mathrm{BIP}_{n}$.

For the case $n=8$, we were unable to obtain a complete description using PORTA, even after using the above simplifications, along with several decomposition 'tricks' along the lines of those described in [5]. We obtained a partial description using three procedures.

First, observe that, if an inequality induces a facet of $\mathrm{CUT}_{n}$ and is valid for $\mathrm{BIP}_{n}$, then it induces a facet of $\mathrm{BIP}_{n}$. So, we took the conjectured complete description of $\mathrm{CUT}_{8}$, available online at [6], and identified the inequalities that met this condition. In fact, 147 equivalence classes are listed on the site, where two inequalities are deemed to be equivalent if one can be obtained from the other either by a permutation of the nodes, or by the so-called switching operation (see [2] or [9] for a formal definition of switching). It turned out that, in 72 out of 147 cases, the inequality could be switched to make it valid for $\mathrm{BIP}_{8}$. This yielded 72 facet classes for $\mathrm{BIP}_{8}$.

Second, we use the (easy) result that, if an inequality of the form

$\sum_{1 \leq i<j \leq n-1} \alpha_{i j} x_{i j} \leq \beta$

induces a facet of $\mathrm{BIP}_{n-1}$, then it also induces a facet of $\mathrm{BIP}_{n}$. Using this result, together with our complete description of $\mathrm{BIP}_{7}$, we were able to obtain an additional eight facet classes for BIP 8 .

Finally, we used some results of Barahona et al. [3], which enable one to obtain facets of $\mathrm{BIP}_{n}$ from facets of $\mathrm{BIP}_{n-2}$. We found that, by taking one particular facet of $\mathrm{BIP}_{6}$, and applying these operations, we were able to derive five additional facet classes of $\mathrm{BIP}_{8}$. That makes 85 facet classes in total.

\section{An annotated list of facets}

Table 1 gives a summary of the results for $n \leq 7$. In this table, we present only one representative of each equivalence class, where two inequalities are deemed to be equivalent if one can be obtained from the other by a permutation of nodes. (There is no switching symmetry for BIP $_{n}$.) Moreover, for any given $n$, we do not present an inequality for $\mathrm{BIP}_{n}$ if it can be derived trivially from an inequality for $\mathrm{BIP}_{n-1}$, as explained in the penultimate paragraph of the previous section.

We now make some remarks on the inequalities listed in the table:

- We let $C_{t}$, for $t \geq 3$ and odd, denote an odd circuit inequality of the form (1), where $t=|C|$. These inequalities appeared in [3].

- We let $K_{t}$, for $t \geq 3$ and odd, denote an 'odd clique' inequality of the form

$\sum_{\{i, j\} \subset S} x_{i j} \leq\left\lfloor|S|^{2} / 4\right\rfloor$,

where $S \subset V$ and $t=|S|$. These inequalities also appeared in [3].

- The term 'hyp', followed by a vector $b \in \mathbb{Z}^{n}$, indicates that the inequality can be obtained by applying the switching operation to a hypermetric inequality of the form

$$
\sum_{1 \leq i<j \leq n} b_{i} b_{j} x_{i j} \leq 0
$$

where $\sum_{1 \leq i \leq n} b_{i}=1$. See [9] for an extensive survey on hypermetric inequalities.

- The subdivided $K_{5}$ and split $K_{5}$ inequalities can be obtained from $K_{5}$ inequalities by applying the operations in [3], mentioned at the end of the previous section.

- The bicycle wheel inequalities were also introduced in [3].

- The clique web inequalities, introduced by Deza and Laurent [8], form a huge class of valid inequalities for $\mathrm{CUT}_{n}$. For the sake of brevity, we do not present the general formula for them here.

- The inequalities labelled 'NEW' do not seem to have appeared in the literature before now.

We remark that, whereas the $K_{t}$, hypermetric, bicycle wheel and clique web inequalities induce facets also of $\mathrm{CUT}_{n}$, the remaining inequalities (lower and upper bounds, $C_{5}, C_{7}$, subdivided $K_{5}$, split $K_{5}$, NEW1 and NEW2) do not.

For the sake of brevity, we do not present the list of facets of $\mathrm{BIP}_{8}$ here. We have however made our results, for $n$ up to 8, available on the web [6].

\section{Computing integrality ratios}

In this section, we show that linear descriptions of $\mathrm{BIP}_{n}$ can be used to compute integrality ratios of various relaxations of the maxcut problem with non-negative edge weights.

For a given value of $n$, let $T_{n}$ be any convex subset of $\mathbb{R}\left(\begin{array}{l}n \\ 2\end{array}\right)$ that is known to contain $\mathrm{CUT}_{n}$. We will call $T_{n}$ a relaxation of $\mathrm{CUT}_{n}$. Such a relaxation could be defined using, for example, linear or semidefinite programming. To evaluate the quality of a relaxation, we are interested in the worst possible ratio, over all instances with non-negative edge weights, between the upper bound obtained by optimising over $T_{n}$, and the weight of an optimal cut. That is, we wish to compute the following quantity:

$$
\sup _{\left.w \in \mathbb{R}_{+}^{(} \begin{array}{c}
n \\
2
\end{array}\right)} \frac{\max \left\{w^{T} x: x \in T_{n}\right\}}{\max \left\{w^{T} x: x \in \mathrm{CUT}_{n}\right\}},
$$

where by convention $0 / 0=1$. This quantity is called the integrality ratio of $T_{n}$.

Now observe that the numerator in (4) is unchanged if we replace $T_{n}$ with its submissive, i.e., with the convex set

$\tilde{T}_{n}=\left\{x \in \mathbb{R}_{+}^{\left(\begin{array}{c}n \\ 2\end{array}\right)}: x \leq x^{\prime}\right.$ for some $\left.x^{\prime} \in T_{n}\right\}$.

Similarly, the denominator in (4) is unchanged if we replace $\mathrm{CUT}_{n}$ with $\operatorname{BIP}_{n}$. Note also that $\tilde{T}_{n}$ and $\operatorname{BIP}_{n}$ are so-called antiblocking 
Table 1

Facets of $\mathrm{BIP}_{n}$, for small values of $n$.

\begin{tabular}{|c|c|c|}
\hline Nodes & Inequality & Type \\
\hline \multirow[t]{2}{*}{2} & $x_{12} \geq 0$ & Lower bound \\
\hline & $x_{12} \leq 1$ & Upper bound \\
\hline 3 & $x_{12}+x_{13}+x_{23} \leq 2$ & $C_{3}=K_{3}$ \\
\hline \multirow[t]{2}{*}{5} & $x_{12}+x_{23}+x_{34}+x_{45}+x_{15} \leq 4$ & $C_{5}$ \\
\hline & $x_{12}+x_{13}+x_{14}+x_{15}+x_{23}+x_{24}+x_{25}+x_{34}+x_{35}+x_{45} \leq 6$ & $K_{5}$ \\
\hline 6 & $2 x_{12}+2 x_{13}+2 x_{14}+2 x_{15}+2 x_{16}+x_{23}+x_{24}+x_{25}+x_{26}+x_{34}+x_{35}+x_{36}+x_{45}+x_{46}+x_{56} \leq 12$ & $\operatorname{hyp}(2,1,1,-1,-1,-1)$ \\
\hline \multirow[t]{11}{*}{7} & $x_{12}+x_{23}+x_{34}+x_{45}+x_{56}+x_{67}+x_{17} \leq 6$ & $C_{7}$ \\
\hline & $x_{13}+x_{14}+x_{15}+x_{16}+x_{23}+x_{24}+x_{25}+x_{27}+x_{34}+x_{35}+x_{45}+x_{67} \leq 8$ & Subdivided $K_{5}$ \\
\hline & $x_{12}+x_{13}+x_{14}+x_{15}+x_{16}+x_{17}+x_{23}+x_{24}+x_{25}+x_{26}+x_{27}+x_{34}+x_{37}+x_{45}+x_{56}+x_{67} \leq 10$ & Bicycle wheel \\
\hline & $2 x_{12}+2 x_{13}+x_{24}+x_{25}+x_{36}+x_{37}+x_{45}+x_{46}+x_{47}+x_{56}+x_{57}+x_{67} \leq 10$ & Split $K_{5}$ \\
\hline & $\begin{aligned} x_{12} & +x_{13}+x_{14}+x_{15}+x_{16}+x_{17}+x_{23}+x_{24}+x_{25}+x_{26}+x_{27}+x_{34}+x_{35}+x_{36}+x_{37} \\
& +x_{45}+x_{46}+x_{47}+x_{56}+x_{57}+x_{67} \leq 12\end{aligned}$ & $K_{7}$ \\
\hline & $\begin{array}{l}x_{12}+x_{13}+x_{14}+x_{15}+x_{16}+x_{17}+x_{23}+x_{24}+x_{25}+x_{26}+x_{27}+x_{34}+x_{35}+x_{36}+x_{37} \\
\quad+x_{45}+x_{46}+x_{47}+2 x_{67} \leq 12\end{array}$ & NEW1 \\
\hline & $\begin{array}{l}x_{13}+x_{14}+x_{15}+x_{16}+2 x_{17}+x_{23}+x_{24}+x_{25}+2 x_{26}+x_{27}+x_{34}+x_{35}+2 x_{36}+2 x_{37} \\
\quad+x_{45}+2 x_{46}+2 x_{47}+2 x_{56}+2 x_{57}+3 x_{67} \leq 18\end{array}$ & Clique web 1 \\
\hline & $\begin{array}{l}x_{12}+x_{13}+x_{14}+x_{15}+2 x_{16}+x_{23}+x_{24}+x_{25}+2 x_{27}+2 x_{34}+2 x_{35}+2 x_{36}+2 x_{37} \\
\quad+2 x_{45}+2 x_{46}+2 x_{47}+2 x_{56}+2 x_{57} \leq 18\end{array}$ & NEW2 \\
\hline & $\begin{array}{l}4 x_{12}+2 x_{13}+2 x_{14}+2 x_{15}+2 x_{16}+2 x_{17}+2 x_{23}+2 x_{24}+2 x_{25}+2 x_{26}+2 x_{27}+x_{34}+x_{35}+x_{36} \\
\quad+x_{37}+x_{45}+x_{46}+x_{47}+x_{56}+x_{57}+x_{67} \leq 20\end{array}$ & $\operatorname{hyp}(2,2,1,-1,-1,-1,-1)$ \\
\hline & $\begin{array}{l}3 x_{12}+3 x_{13}+3 x_{14}+3 x_{15}+3 x_{16}+3 x_{17}+x_{23}+x_{24}+x_{25}+x_{26}+x_{27}+x_{34}+x_{35}+x_{36} \\
\quad+x_{37}+x_{45}+x_{46}+x_{47}+x_{56}+x_{57}+x_{67} \leq 20\end{array}$ & $\operatorname{hyp}(3,1,1,-1,-1,-1,-1)$ \\
\hline & $\begin{aligned} x_{12} & +x_{13}+x_{14}+2 x_{15}+2 x_{16}+3 x_{17}+x_{23}+x_{24}+2 x_{25}+2 x_{26}+3 x_{27}+x_{34}+2 x_{35}+2 x_{36}+3 x_{37} \\
& +2 x_{45}+2 x_{46}+3 x_{47}+3 x_{56}+5 x_{57}+5 x_{67} \leq 28\end{aligned}$ & Clique web 2 \\
\hline
\end{tabular}

sets, which means that they belong to the non-negative orthant and coincide with their own submissive.

We now recall the following result from [11]:

Proposition 1 (Goemans and Hall). Let $Q \subset \mathbb{R}_{+}^{d}$ be a polytope of antiblocking type and let $P \subset \mathbb{R}_{+}^{d}$ be a convex set of antiblocking type that contains $Q$. Assume that

$Q=\left\{x \in \mathbb{R}_{+}^{d}: a_{i} x \leq b_{i}\right.$ for $\left.i=1, \ldots, m\right\}$,

where $a_{i}$ and $b_{i}$ are non-negative for $i=1, \ldots, m$. Then,

$\sup _{w \in \mathbb{R}_{+}^{d}} \frac{\max \left\{w^{T} x: x \in P\right\}}{\max \left\{w^{T} x: x \in Q\right\}}=\max _{1 \leq i \leq m} \frac{\max \left\{a_{i} x: x \in P\right\}}{b_{i}}$.

(In fact, Goemans and Hall proved this result only for the case in which $P$ is a polytope, but the proof holds for general convex sets $P$.)

Setting $P=\tilde{T}_{n}$ and $Q=\operatorname{BIP}(n)$ in Proposition 1 , we arrive at the following result:

Corollary 1. For a given $n$, let $\mathcal{F}$ denote the set of facets of $\mathrm{BIP}_{n}$ that are not defined by non-negativity inequalities. Let the corresponding facet-defining inequalities be denoted by $\alpha^{i} \cdot x \leq \beta_{i}$, for $i \in \mathcal{F}$. Then the integrality ratio (4) is equal to

$\max _{i \in \mathcal{F}} \frac{\max \left\{\alpha^{i} \cdot x: x \in T_{n}\right\}}{\beta_{i}}$.

In this way, we reduce the computation of the integrality ratio to a finite series of optimisation problems. Moreover, we can reduce the amount of work needed by exploiting the symmetry of $\mathrm{BIP}_{n}$. Specifically, instead of solving one optimisation problem for each inequality defining a facet of $\mathrm{BIP}_{n}$, it suffices to solve one optimisation problem for each equivalence class, where, as in the previous section, two inequalities are deemed to be equivalent if one can be obtained from the other by a permutation of nodes.

\section{An annotated table of ratios}

Table 2 presents the integrality ratios that we obtained, for several different relaxations of the max-cut problem, and for several values of $n$. For convenience, we present the ratios as both fractions and decimals, where possible. (The ratio denoted by ' $\phi\left(C_{5}\right)$ ' is an irrational number, as explained below.) The values presented for $n \leq 7$ are exact. The column for $n=8$ is marked with an asterisk because the values are based on our partial linear description of $\mathrm{BIP}_{8}$, and therefore represent lower bounds on the true value. Nevertheless, we conjecture that these values are also exact.

The following relaxations appear in the table:

- The 'trivial' relaxation, defined by the bounds $0 \leq x_{e} \leq 1$ for all $e \in E$.

- The 'triangle' relaxation, which is the linear relaxation defined by the triangle inequalities:

$$
\begin{array}{ll}
x_{i j}+x_{i k}+x_{j k} \leq 2 & (1 \leq i<j<k \leq n), \\
x_{i j}-x_{i k}-x_{j k} \leq 0 & (1 \leq i<j \leq n ; k \neq i, j) .
\end{array}
$$

- The 'odd clique' relaxation, which is the linear relaxation defined by all inequalities that can be derived from the odd clique inequalities (2) by switching.

- The 'hypermetric' relaxation, which is the linear relaxation defined by all inequalities that can be derived from the hypermetric inequalities (3) by switching.

- The 'sdp' relaxation, which is the semidefinite relaxation derived by Schrijver (unpublished) and used in the famous approximation algorithm of Goemans and Williamson [12].

- Three further relaxations obtained by combining the previous linear and semidefinite relaxations.

That makes eight relaxations in total.

As explained in the previous section, to compute each number in Table 2, a sequence of linear or semidefinite programs had to be 
Table 2

Integrality ratios of various relaxations of the max-cut problem, for small values of $n$. For a definition of $\phi\left(C_{5}\right)$, see the text.

\begin{tabular}{lllllll}
\hline$n$ & 3 & 4 & 5 & 6 & 7 & $8^{*}$ \\
\hline Trivial & $3 / 2$ & $3 / 2$ & $5 / 3$ & $5 / 3$ & $7 / 4$ & $7 / 4$ \\
Triangle & 1.5 & 1.5 & 1.667 & 1.667 & 1.75 & 1.75 \\
& 1 & 1 & $10 / 9$ & $10 / 9$ & $7 / 6$ & $7 / 6$ \\
Odd clique & 1 & 1 & 1.111 & 1.111 & 1.167 & 1.167 \\
& 1 & 1 & 1 & $25 / 24$ & $21 / 20$ & $21 / 20$ \\
Hypermetric & 1 & 1 & 1 & 1.042 & 1.05 & 1.05 \\
& 1 & 1 & 1 & 1 & $31 / 30$ & $31 / 30$ \\
sdp & 1 & 1 & 1 & 1 & 1.033 & 1.033 \\
sdp + triangle & 1 & 1 & $25 / 24$ & $25 / 24$ & $47 / 45$ & $47 / 45$ \\
& 1 & 1 & 1.042 & 1.042 & 1.044 & 1.044 \\
sdp + odd clique & 1 & 1 & 1 & $49 / 48$ & $31 / 30$ & $31 / 30$ \\
& 1 & 1 & 1 & 1.021 & 1.033 & 1.033 \\
sdp + hypermetric & 1 & 1 & 1 & 1 & $31 / 30$ & $31 / 30$ \\
& 1 & 1 & 1 & 1 & 1.033 & 1.033 \\
& 1 & 1.125 & 1.131 & 1.131 & 1.131 & 1.131 \\
& 1 & 1 & & & &
\end{tabular}

solved. To this end, we had to implement a cutting-plane algorithm for small max-cut instances. Note that the number of switched odd clique and hypermetric inequalities grows exponentially with $n$, and their corresponding separation problems had to be solved exactly in order to obtain reliable ratios. Fortunately, for such small values of $n$, it was possible to solve the separation problems exactly by integer programming. For the sake of brevity, we do not describe the integer programming formulations in detail.

To aid the reader, we now describe the specific max-cut instances for which each of the ratios in Table 2 are attained:

- The ratios $3 / 2,5 / 3$ and $7 / 4$ in the 'trivial' row arise when the objective function is equal to the left-hand side (LHS) of a $K_{3}, K_{5}$ and $K_{7}$ inequality, respectively.

- The ratios 10/9 and 7/6 in the 'triangle' row arise when the objective function is equal to the LHS of a $K_{5}$ and $K_{7}$ inequality, respectively.

- The ratios $25 / 24$ and $21 / 20$ in the 'odd clique' row arise when the objective function is the LHS of a switched hypermetric inequality with $b=(2,1,1,-1,-1,-1)$ and $b=(3,1,1$, $-1,-1,-1,-1)$, respectively.

- The ratio 31/30 which appears in the 'hypermetric', 'sdp + odd clique' and 'sdp + hypermetric' rows arises when the objective function is the LHS of a bicycle wheel inequality.

- The ratio 9/8 in the 'sdp' row arises when the objective function is the LHS of a $K_{3}$ inequality.

- The ratio denoted by ' $\phi\left(C_{5}\right)$ ' in the 'sdp' row arises when the objective function is the LHS of a $C_{5}$ inequality. Delorme and Poljak [7] showed that $\phi\left(C_{5}\right)=(25+5 \sqrt{5}) / 32$.

- The ratios of $25 / 24$ and $47 / 45$ in the 'sdp + triangle' row arise when the objective function is the LHS of a $K_{5}$ and bicycle wheel inequality, respectively.

- Finally, the ratio of $49 / 48$ in the 'sdp + odd clique' row arises when the objective function is the LHS of a switched hypermetric inequality with $b=(2,1,1,-1,-1,-1)$.
We remark that, as $n$ goes to infinity, the integrality ratio approaches 2 for the 'trivial' and 'triangle' relaxations [18], and

$\frac{\pi}{2} \max _{0 \leq \theta \leq \pi} \frac{1-\cos \theta}{\theta} \approx 1.1383$

for the 'sdp' and 'sdp + triangle' relaxations (see $[10,16]$, respectively). To our knowledge, the corresponding figures for the other relaxations are unknown.

\section{Acknowledgement}

The second author was supported by the Engineering and Physical Sciences Research Council under grant EP/D072662/1.

\section{References}

[1] F. Barahona, On the complexity of max-cut, Research report no. 186, IMAG Université Scientifique et Medicale de Grenoble, France, 1980.

[2] F. Barahona, A.R. Mahjoub, On the cut polytope, Math. Program. 36 (1986) 157-173.

[3] F. Barahona, M. Grötschel, A.R. Mahjoub, Facets of the bipartite subgraph polytope, Math. Oper. Res. 10 (1985) 340-358.

[4] T. Christof, A. Loebl, PORTA (polyhedron representation transformation algorithm). Software package, available for download at http://www.zib.de/ Optimization/Software/Porta/.

[5] T. Christof, G. Reinelt, Decomposition and parallelization techniques for enumerating the facets of combinatorial polytopes, Int. J. Comput. Geom. Appl. 11 (2001) 423-437.

[6] T. Christof, G. Reinelt, SMAPO (library of small polytopes related to combinatorial optimization problems). Available for download at http://comopt.ifi.uniheidelberg.de/software/SMAPO.

[7] C. Delorme, S. Poljak, Laplacian eigenvalues and the maximum cut problem, Math. Program. 62 (1993) 557-574.

[8] M.M. Deza, M. Laurent, Facets for the cut cone (parts I and II), Math. Program. 56 (1992) 121-188

[9] M.M. Deza, M. Laurent, Geometry of Cuts and Metrics, Berlin, Springer-Verlag, 1997.

[10] U. Feige, G. Schechtman, On the optimality of the random hyperplane rounding technique for max cut, Random Struct. Algorithms 20 (2002) 403-440.

[11] M.X. Goemans, L.A. Hall, The strongest facets of the acyclic subgraph polytope are unknown, in: W.H. Cunningham, S.T. McCormick, M. Queyranne (Eds.), Integer Programming and Combinatorial Optimization V, in: Lecture Notes in Computer Science, vol. 1084, Springer, Berlin, 1996, pp. 415-429.

[12] M.X. Goemans, D.P. Williamson, Improved approximation algorithms for maximum cut and satisfiability problems using semidefinite programming, J. Assoc. Comput. Mach. 42 (1995) 1115-1145.

[13] V.P. Grishukhin, All facets of the cut cone for $n=7$ are known, Eur. J. Comb. 11 (1990) 115-117.

[14] M. Grötschel, W.R. Pulleyblank, Weakly bipartite graphs and the max-cut problem, Oper. Res. Lett. 1 (1981) 23-27.

[15] R. Karp, Reducibility among combinatorial problems, in: R. Miller, J. Thatcher (Eds.), Complexity of Computer Computations, Plenum Press, New York, 1972, pp. 85-103.

[16] S.A. Khot, N.K. Vishnoi, The unique games conjecture, integrality gap for cut problems and embeddability of negative type metrics into $\ell_{1}$, in: Proc. of the 46th Annual IEEE Symposium on Foundations of Computer Science, 2005 pp. 53-62.

[17] M. Laurent, Max-cut problem, in: M. Dell'Amico, F. Maffioli, S. Martello (Eds.), Annotated Bibliographies in Combinatorial Optimization, Wiley, Chichester 1997, pp. 241-259.

[18] S. Poljak, Zs. Tuza, The expected relative error of the polyhedral approximation of the max-cut problem, Oper. Res. Lett. 16 (1994) 191-198.

[19] S. Poljak, Zs. Tuza, Maximum cuts and large bipartite subgraphs, in: W. Cook L. Lovász, P. Seymour (Eds.), Combinatorial Optimization, in: DIMACS Series in Discrete Mathematics and Theoretical Computer Science, vol. 20, American Mathematical Society, 1995, pp. 181-244. 